\title{
Precision Reference Specimens of Surface Roughness: Some Characteristics of the Cali-Block*
}

\author{
Russell D. Young and Fredric E. Scire \\ Institute for Basic Standards, National Bureau of Standards, Washington, D.C. 20234
}

(March 27, 1972)

\begin{abstract}
The distribution of Arithmetic Average values of surface roughness across the 120 and 20 microinch patches of NBS Cali-Block " $B$ " have been measured. It is shown that each distribution can be described in terms of its mean value and standard deviation. The observed systematic distribution of $A A$ values strongly suggests that the user select several widely spaced regions at random on the patch when calibrating a roughness measuring instrument.
\end{abstract}

Key words: Arithmetic Average roughness; Cali-Block; calibration; roughness.

\section{Introduction}

The American Standard for surface finish measurement (ANSI B46.1-1962) specifies the precision reference specimen for calibrating surface finish instrumentation as follows (in part): "The normal surface profile of Precision Reference Specimens of roughness height shall consist of a series of isosceles triangles having included angle of $150^{\circ}$ " - "Roughness values shall not vary from the designated value by more than 1 microinch or 3 percent, whichever is larger."

The Natonal Bureau of Standards (NBS) maintains several carefully calibrated precision reference specimens which are employed as calibration masters in the comparison procedure used in surface finish calibration services. Most of the calibration load involves a U.S. manufactured specimen called the Cali-Block, which consists of two patches, one with a nominal Arithmetic Average (AA) roughness of 125 microinches and another with 20 microinches. The corresponding standard maintained by NBS consists of a Cali-Block whose $A A$ roughness was determined by: (1) calculating the area under a carefully measured profile of the surface, and (2) calibrating the surface profile measuring instrument with an interferometrically measured step. The groove shape employed in the Cali-Block conforms to ANSI B46.1. The NBS standard Cali-Block was calibrated, as described

*Certain commercial equipment, instruments, or materials are identified in this paper in order to adequately specify the experimental procedure. In no case does such identification imply recommendation or endorsement by the National Bureau of Standards, no does it imply that the material or equipment identified is necessarily the best available for the purpose. above, by three laboratories in a blind round robin. Each of the three values fell well within the $3 \sigma$ limit of the other two laboratories. The average of these three measurements is defined as the roughness of the maintained standard. NBS continually examines and reevaluates the measurement process associated with its many calibration services. As a part of this program, we have investigated the distribution of $A A$ values over the surface of the two Cali-Block patches on NBS Cali-Block "B", whose history is well known. Briefly, Cali-Block "B" was calibrated several years ago and was then set aside as a "spare" and was essentially unused. It was kept in a protective container in the intervening period. After assuring the cleanliness of the patches, the following measurements were carried out on this block. Since the results obtained were similar to those encountered in previous Cali-Block measurements, it is assumed that the following results are typical for Cali-Blocks. This conclusion is supported by the fact that Cali-Blocks are manufactured by an electrodeposition replicating process which should produce a family of almost identical members.

\section{Procedure}

The Cali-Block was mounted on an $X-Y$ table in a stylus instrument. The first measurement of surface roughness was carried out in one corner of the 125 microinch patch by making two standard 30 mil scans. The reproducibility of the measurement was such that these two readings were either identical or well within 1 percent of each other. The block was then advanced 
approximately $3 \mathrm{~mm}$ and the process repeated. In this fashion each $3 \mathrm{~mm}$ by $2 \mathrm{~mm}$ square area on the patch surface was measured. A total of 180 areas were thus measured on each of the two patches. The results are displayed on the $A A$ profile map in figure 1. A similar map for the 20 microinch patch is shown in figure 2 .

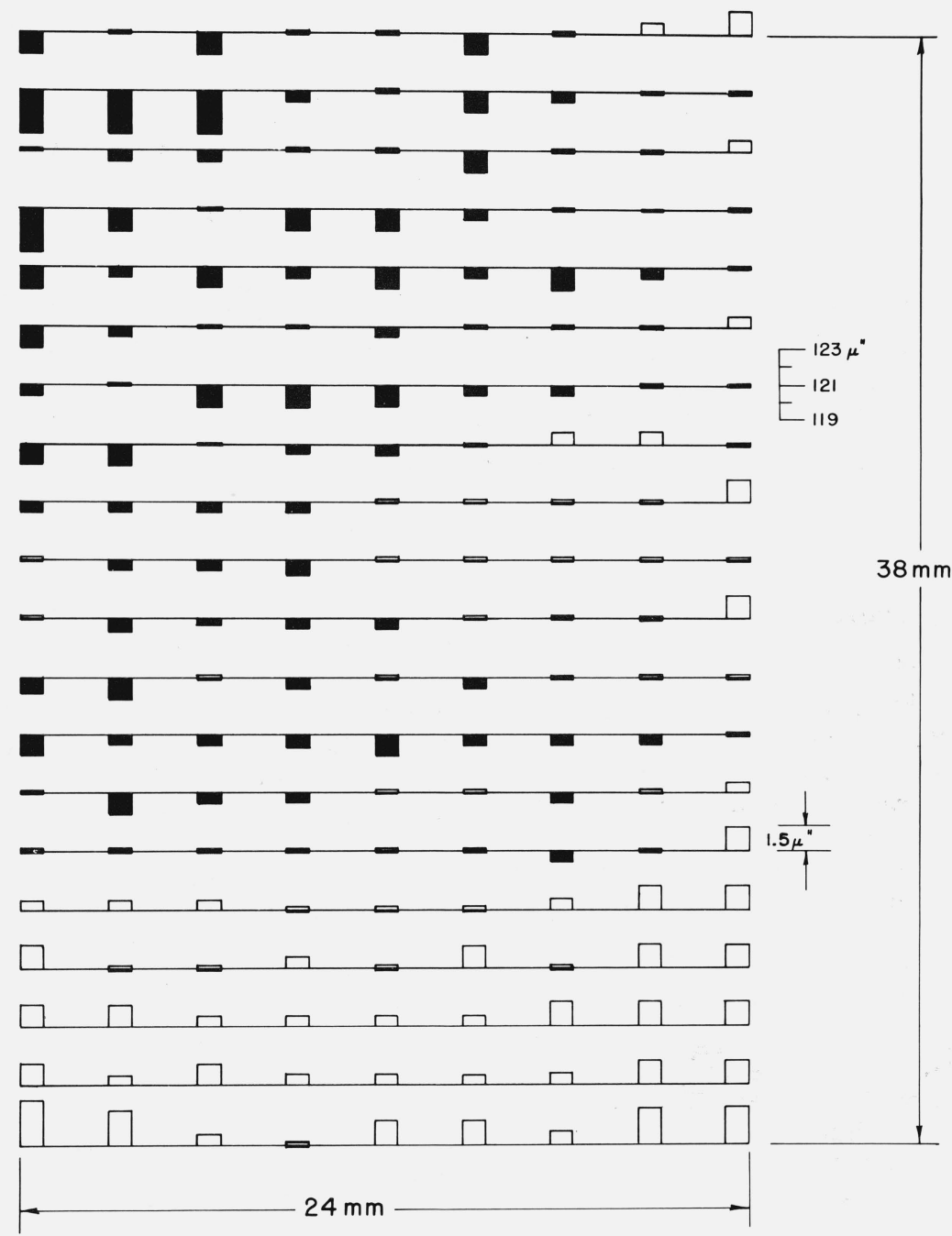

FIGURE 1. Distribution of Arithmetic Average (AA) roughness values over the surface of the 120 microinch patch of Cali-Block.

Each of the 88 black (or white) boxes represents the amount by which the $A A$ value deviated from the mean of the $A A$ values for all 88 measurements. Locations where the $A A$ value exceeded the mean value are depicted by white bars while smaller values are depicted by black bars. 


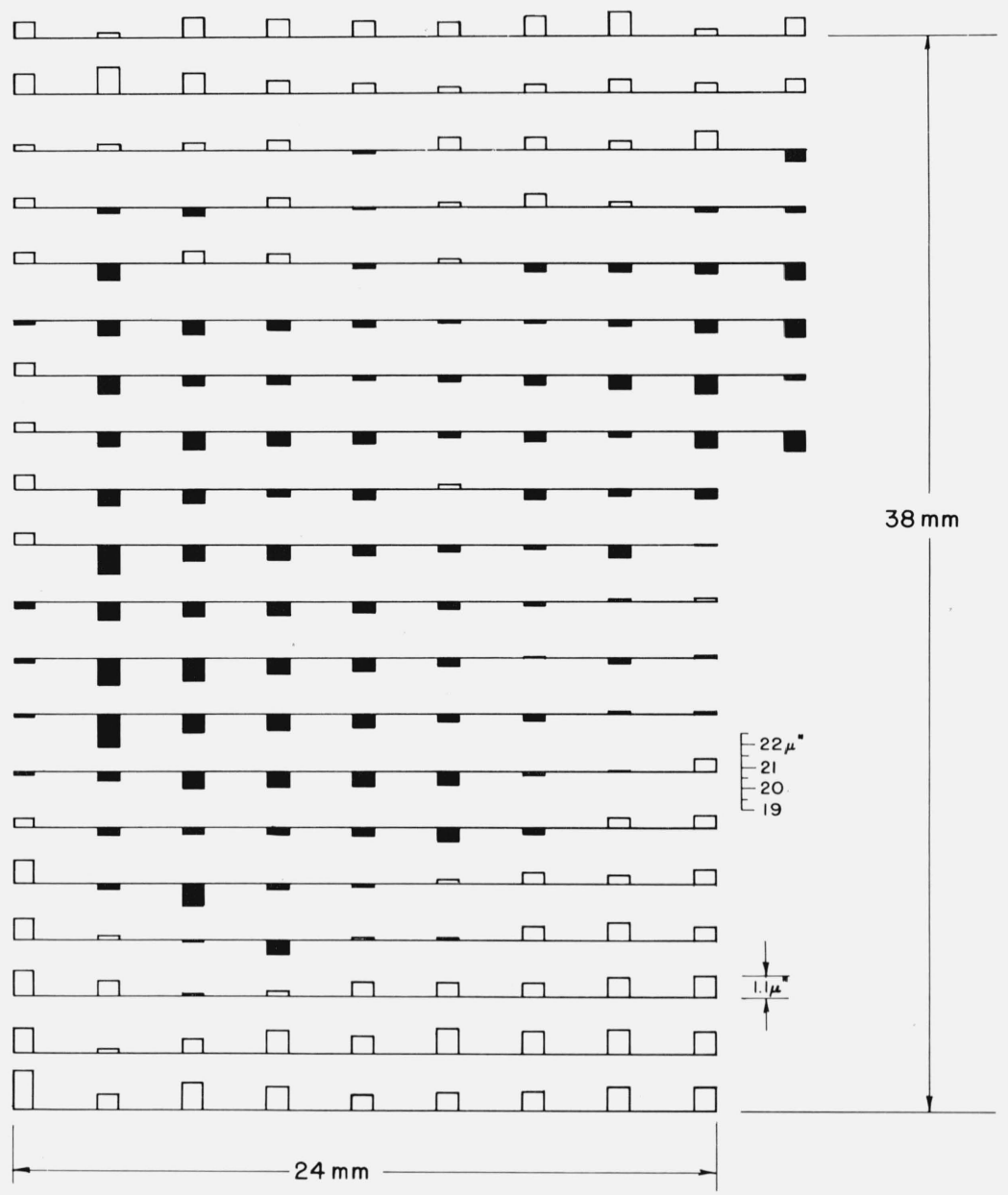

Figure 2. Distribution of AA values over the surface of the 20 microinch patch of Cali-Block B, as in figure 1 .

\section{Discussion of Results}

It would be particularly useful to find a way in which the results in figures 1 and 2 can be succinctly characterized. If the deviations of the $A A$ values from the mean value are random, then the mean value, standard deviation and $3 \sigma$ values should provide a valuable description of the $A A$ roughness distribution over the patches.

It is clear from figures 1 and 2 that there is a systematic variation in $A A$ values over the surface of each patch. However, about 68 percent of the measurements were within one $\sigma$ of the mean and only one value $(0.5 \%)$ exceeded the mean by $3 \sigma$. It is anticipated (and recommended) that the user will select several widely spaced regions at random on the patch when calibrating his roughness measuring instrument. This reduces patch wear and serves to minimize the effect of the systematic variation in $A A$ values on the accuracy of the calibration. Assuming such a random selection of regions, the standard deviation should provide a useful description of the measurement process. Such a characterization is particularly useful since the techniques for combining $\sigma$ values with other uncertainties, such as random human errors and instrumental errors, are well known. The results are reported in table 1 .

TABLE 1. Mean roughness, standard deviation $(\sigma)$ and $3 \sigma$ values for each of the ${ }_{i}$ )atches on NBS Cali-Block " $B$ "'

\begin{tabular}{c|c|c|c}
\hline \hline Patch & $\begin{array}{c}\text { Mean value } \\
A A \text { roughness }\end{array}$ & $\sigma$ & $3 \sigma$ \\
\hline $\begin{array}{r}125 \text { microinches } \\
20 \text { microinches }\end{array}$ & $\begin{array}{r}120.9 \\
20.8\end{array}$ & $\begin{array}{r}0.70 \\
0.66\end{array}$ & $\begin{array}{c}2.1 \text { microinches. } \\
2.0 \text { microinches. }\end{array}$ \\
\hline
\end{tabular}

\section{Conclusion}

It is evident from this study that one important limiting factor in the accuracy and precision with which surface roughness standards can be transmitted through the use of the Cali-Block is the variation in $A A$ value across the surface of the patch. Furthermore, the importance of a random selection of several widely spaced regions on the patch during calibration is very evident.

(Paper 76C1 \&2-328) 